\title{
The Transcendental Aesthetic and Absolute Totality of Conditions: The Problem of Metaphysics in the Critique of Pure Reason and a Solution Kazuhiko YAMAMOTO \\ Kyushu University, Faculty of Arts and Sciences, Fukuoka, Japan
}

\begin{abstract}
Kant's metaphysics, which says that the absolute whole of magnitude - the world-whole - has nothing to do with any possible experience, presses him to think of a thing in itself, which is merely intelligible. The difficulty is related to the issue of the absolute totality of series of conditions in connection with the issue of the absolute magnitude of the series in the world of sense, which looms as the antinomy of pure reason in the system of cosmological ideas. Since pure reason has no other aim than the absolute totality of synthesis on the side of conditions, we have to take steps in order to solve this problem, namely the absolute totality of synthesis on the side of conditions in conjunction with the absolute magnitude of the series in the world of sense, in such a way that we can comprehend transcendental aesthetics and the world-whole through empirical intuition and synthesis in accordance with experience or possible experience.
\end{abstract}

Keywords: Transcendental Aesthetics, Intuition, Possible Experience, Absolute Totality

\section{INTRODUCTION}

Kant, who firmly believes that the universal principles of ethics have to flow merely from his concepts of reason (A480/B508), launches an experiment which is expected to prove the pure principles of reason: "that absolutely no concepts" that "contain anything empirical" must enter into "the complete estimation of synthetic a priori cognition," or "that the a priori cognition be entirely pure" (A14/B28). In performing this experiment, Kant cannot ground the transcendental concept of reason, namely the concept of "the totality of conditions to a given conditioned thing" (B379), and "the pure categories," which, Kant thinks, are erroneously assumed to be within "the field of possible experience" (A248-B305), in a world of sense through understanding. When Kant, being trapped in the dilemma, divulges a direction of his ways of thinking (B379), we take it as a hint, which would lead us to the finding that that which is merely in us can "determine the constitution of an object distinct from our representations, i.e., be a ground why there should be a thing that corresponds to something we have in our thought, and why all this representation should not instead be empty" (A129). We understand that this scheme signifies Kant's "synthetic a priori cognition" (YAMAMOTO 2016: 87-100). The criterion of the possibility of the concept of object in itself death itself - is not the definition but the necessity which "lies solely in the law of possible experience that everything that happens is determined a priori through its cause in appearance" (B280) - the law of nature (YAMAMOTO 2016: 87-100). Kant's difficulty in his metaphysics is related to the issue of "the absolute totality of series of conditions in the world of sense," in connection with the "issue about the absolute magnitude of the series in this world" (A515/B543-A516/B544), which looms as the antinomy of pure reason in the system of cosmological ideas. Since "pure reason has no other aim than the absolute totality of synthesis on the side of conditions," and "reason has nothing to do with absolute completeness from the side of the conditioned" (A336), we have to take a next step to solve this difficult problem through attaining "the definition, in which the unity of the concept, the truth of everything that may initially be derived from" empirical cognition, and "finally the completeness of everything that is drawn from it," are to "constitute everything that is necessary for the production of the entire concept" (B115).

\section{TransCendental AesthetiC AND Synthesis OF APPHERENSION}

Kant, who thinks the representation 'I think' to be the topos for the connection of all representations, but not the condition of it, has to say that time "contains an a priori manifold in pure intuition," which 
is equivalent to the transcendental time-determination (A138/B177). Thinking that this " $a$ priori manifold in pure intuition" was nothing but illusion in his brain, we have clarified that the transcendental time-determination signifies nullity in time - time itself, which is not an illusion but has content as death itself (YAMAMOTO 2016: 87-100). We have to think that since "the standing and lasting I of pure apperception" (A123) constitutes the "true correlate" (A30) of all of our representations - "the thing in itself" (A30) - "just as all sensible intuition as representation belongs to a pure inner intuition, namely that of time" (A123-A124), this inner intuition - time - is nullified when 'the standing and lasting I of pure apperception' is utterly dismembered upon disappearing under the law of nature. In regard to time, Kant explicates it, saying: "The infinitude of time signifies nothing more than that every determinate magnitude of time is only possible through limitations of a single time grounding it. The original representation, time, must therefore be given as unlimited. But where the parts themselves and every magnitude of an object can be determinately represented only through limitation, there the entire representation cannot be given through concepts (for then the partial representations precede) but their immediate intuition must be the ground" (A32-B48). What he refers to seems to indicate three things: 1) "the infinitude of time" signifies "the original representation, time" - time itself - which is unlimited, thereby "a single time:" 2) "every determinate magnitude of time," which becomes possible through limitations of a single time, is homogeneous with "the infinitude of time" - time itself: 3) time itself and "every determinate magnitude of time" elapsing time - are grounded on "their immediate intuition." We have already found that 1) absolute disappearance of a human, namely death itself, which necessarily and universally occurs, signifies nullity in space-time: 2) appearance itself signifies filled space-elapsing time or empty space-nullified time as "every empirical representation of the manifold" (A139): 3) nullity in space-time permeates filled space-elapsing time (YAMAMOTO 2016: 87-100). In light of this finding, we say that 1) in regard to time, a singularity appears in elapsing time: 2) elapsing time is homogeneous with time itself which is limited in 'elapsing.' Thus, a singularity, namely 'the standing and lasting I of pure apperception,' which appears in elapsing time, is to disappear in nullified time (YAMAMOTO 2016: 87-100). It comes out that the determinate magnitude of time in the world of sense signifies 'elapsing time,' while the infinitude of time signifies 'nullified time.' We have to note that 'elapsing time' or 'nullified time' signifies a representation of time and that time itself signifies nullity in time, which is homogeneous with "a thing in itself (A538/B566)." Therefore, we say, in an opposite manner to what Kant says (B46), 'Time is an empirical concept that is somehow drawn from an experience or a possible experience. For simultaneity or succession would, themselves, come into perception since the representation of time did ground them a priori.' When we say that transcendental time-determination is nothing but nullity in time (YAMAMOTO 2016: 87-100), we are saying, following what Kant refers to (A138/B177-B178), that 'this belongs to the category since nullity in time universally permeates a genus and "rests on a rule a priori" - death itself -which is unavoidable according to the law of nature.' Our metaphysics indicates that time, as the condition of the manifold of inner sense, thus of the connection of all representations, contains an a priori manifold in pure intuition, namely nullity in time - the infinitude of time. The transcendental time-determination - nullity in time seems to be homogenous with appearances themselves since it is already contained, as a condition, in every empirical representation of the manifold. The transcendental time-determination constitutes the unity of the category of death itself, indicating that humans, whose unity or schema contains a priori nullity in time, are dying in concurrence with being there as living things.

Our metaphysics rests on whether or not it is possible to cognize, through empirical intuition, if appearance itself is to be necessarily and universally homogeneous with nullity in time - time itself. In other words, is it possible to comprehend, through experience or possible experience, that the condition of the manifold of inner sense, thus of the connection of all representations, is commensurate with nullity in time? What Kant refers to in regard to experience and appearance gives us clues to comprehend time from an alternative viewpoint. Accordingly, when Kant says, "experience teaches us that one appearance customarily follow another, but not that it must necessarily follow that, nor that an inference from a condition to its consequence can be made a priori and entirely universally" (A112), we say that "experience teaches us that disappearance customarily follows one appearance, and that it must necessarily follow that: and that an inference from a condition to its consequence can be made a priori and entirely universally.' What does inference from a condition to its consequence mean? It means that a condition - the disappearance of the standing and lasting I of pure apperception - makes us infer that this disappearance is tantamount to a space being emptied and a time being nullified in regard to that standing and lasting I. In addition, we must say that this inference is invoked as a result of one's own 'possible experience.' Previously, we said 'In the process of disappearing, it becomes impossible for the standing and lasting I of pure apperception to be conscious of all of its representations. As a result, it has to forsake the correlation 
of all of its representations while the other standing and lasting I, in appearance, is to constitute the correlation of representations, as far as it is possible to be conscious of representations of the a priori manifold, namely disappearance. The representations of disappearances are analytically brought by general logic under one concept, which is to be equivalent to the pure concept of the understanding, i.e., death itself. Since the principles of pure understanding can be related to objects of the senses only in relation to the general conditions of a possible experience, but never to things in general (B303), the pure concepts of the understanding are equivalent to 'death,' which humans are destined to come across in possible experience according to the law of nature' (YAMAMOTO 2016: 87-100). Furthermore we said that 'time, as the condition of the manifold of inner sense, thus of the connection of all representations, contains an a priori manifold, namely death, in pure intuition as nullity' (YAMAMOTO 2016: 87-100). Here a serious problem should arise: how do we perceive nullity in time - nullified time - through empirical intuition? Is this perception of nullified time the same as the perception of elapsing time? Again, Kant gives us a hint. He says, "that the empirical rule of association, which one must assume throughout if one says that everything in the series of occurrences stands under rules according to which nothing happens that is not preceded by something upon which it always follows - on what, I ask, does this, as a law of nature, rest, and how is this association even possible? The ground of the possibility of the association of the manifold, insofar as it lies in the object, is called the affinity of the manifold" (A112-A113). From our viewpoint, what he refers to indicates three things: 1) a law of nature rests on the rules that disappearance occurs that is preceded by appearance upon which it always follows, 2) the association of the manifold is possible since its possibility lies in the object in itself, which appears and then disappears, 3) thus, the affinity of the manifold is to be attained through the empirical rule of association, which is under the aegis of the law of nature. In order to make this comprehensible, we have to clarify how nullity in space-time is empirically cognized "in the entirety of all possible experience" (A146), expecting to show that the affinity of the manifold - nullity in space-time - lies in the object in itself as "the possibility of the association of the manifold" (A113).

Before we launch into it, what we have should be clarified in order to cognize nullity in space-time in the entirety of all possible experience. Since transcendental logic "has a manifold of sensibility that lies before it a priori, which the transcendental aesthetic has offered to it, in order to provide the pure concepts of the understanding with a matter" (B102-A77), we have a manifold of sensibility that the transcendental aesthetic has offered. How do we know a manifold of sensibility that the transcendental aesthetic has offered? Kant tries to explicate it, saying "In the transcendental aesthetic we will therefore first isolate sensibility by separating off everything that the understanding thinks through its concepts, so that nothing but empirical intuition remains. Second, we will then detach from the latter everything that belongs to sensation, so that nothing remains except pure intuition and the mere form of appearances, which is the only thing that sensibility can make available a priori" (A22). The enigmatic and contradictory remark seems to suggest that 1) when "everything that the understanding thinks through its concepts" is separated off, what remains is sensibility - empirical intuition: 2) empirical intuition detached from sensation is nothing but "pure intuition and the mere form of appearances:" 3) "the mere form of appearances" is made available a priori through sensibility - empirical intuition. Since the transcendental aesthetic is "a science of all principles of $a$ priori sensibility" (A21), and that "there are two pure forms of sensible intuition as principles of $a$ priori cognition, namely space and time" (A22), we take it as a hint, thinking that 1) "sensibility that lies before it a priori" (B102) is homogeneous with pure intuition affected by the sensation of nullity, 2) when pure intuition is equivalent to empirical intuition from which sensation is detached, pure intuition affected by the sensation of nullity is to be homogeneous with empirical intuition, 3) "pure intuition" (A20-B35) signifies "two pure forms of sensible intuition" - space-time itself, 4) pure intuition affected by the sensation of nullity - empirical intuition - would expose "pure intuition and the mere form of appearances," 5) while pure intuition and the mere form of appearances are a priori, the mere form becomes available a priori through sensibility. With this way of thinking in mind, we should start from sensibility, namely empirical intuition, by exploring "a manifold of sensibility that lies before it a priori, which the transcendental aesthetic has offered to it." In regard to the issue, following what Kant refers to (B240-A195), we say, in an opposite manner, 'in appearance, two states follow one another, that is, a state of disappearance follows a state of appearance.' We think that a state of disappearance is perceived through empirical intuition, which teaches us that 'elapsing time' in regard to 'the standing and lasting I' is nullified, and 'filled space' is emptied. Alteration from filled space-elapsing time to empty space-nullified time - nullity in space-time - occurs necessarily and universally according to the law of nature. When we try to find what empirical intuition means among the standing and lasting Is, we have to know how the standing and lasting Is cognize things "as they 
appear" (A250), through empirical intuition, in the milieu in which geometry or mathematics, as a science, does not wield overwhelming power on the imagination. We think that if geometry or mathematics, as a science, affects the imagination strongly, it might acclimatize empirical intuition along with its power of abstraction since imagination, as "the faculty for representing an object even without its presence in intuition," is "the subjective condition" which might "give a corresponding intuition to the concepts of understanding" (B151). In addition, the imagination which "belongs to sensibility," is thought to be "a faculty for determining the sensibility a priori" to the extent that "its synthesis of intuitions" can be seen as "an exercise of spontaneity" (B151-B152). Since the imagination is thought to be able to "determine the form of sense a priori," it is not far-fetched to assume that if the imagination is affected by geometry or mathematics, with its power of abstraction, empirical intuition is alienated from the imagination - "the faculty for representing an object even without its presence in intuition." We think that when empirical intuition is alienated from the pristine faculty of humans - the imagination - it becomes obscure, being difficult to cognize its true figure through merely examining the manifold of intuition. Does not Kant's view on empirical intuition belong to the Age of Enlightenment, when humans are keenly requested to augment scientific knowledge, property and wealth in material? Is it not affected by the ways of thinking which appreciate being positive (e.g., filled space-elapsing time) more than being negative (e.g., empty space-nullified time)? Here, we hypothesize that 1) imagination is the pristine faculty of humans by virtue of "an exercise of spontaneity," through which it is possible to cognize 'objects as appearance' in filled space-elapsing time, and the disappearance of the 'objects as appearance' in empty space-nullified time, 2) since pure intuition is the empirical intuition from which everything that belongs to sensation is detached, pure intuition affected by the sensation of nullity can be homogeneous with empirical intuition, 3) when humans empirically cognize 'objects as appearance' in filled space-elapsing time, they comprehend it to be space-time which pertains to 'objects as appearance,' 4) filled space-elapsing time - appearance itself - is understood to be "all objects" as "mere appearances" (A49) on account of the fact that the imagination is affected by geometry or mathematics in terms of scientific knowledge. Who understands so? It is Kant himself that understands so. Geometry and mathematics seem to have engrained Kant with a way of thinking, which is that "geometry is a science that determines the properties of space synthetically and yet $a$ priori...from a mere concept no propositions can be drawn that go beyond the concept, which, however, happens in geometry" (B40-B41). Kant refers to geometry or mathematics as follows: "space has only three dimensions; but such propositions cannot be empirical or judgments of experience, nor inferred from them" (B41); "Take the proposition that with two straight lines no space at all can be enclosed, thus no figure is possible, and try to derive it from the concept of straight lines and the number two;...All of your effort is in vain, and you see yourself forced to take refugee in intuition, as indeed geometry always does" (B65-A48); "The synthesis of spaces and times, as the essential form of all intuition, is that which at the same time makes possible the apprehension of the appearance, thus every outer experience, consequently also all cognition of its objects, and what mathematics in its pure use proves about the former is also necessarily valid for the latter. All objections to this are only the chicanery of a falsely instructed reason, which erroneously thinks of freeing the objects of the senses from the formal condition of our sensibility, and, though they are mere appearances, represents them as objects in themselves, given to the understanding; in which case, certainly, nothing synthetic could be cognized of them a priori at all, thus not even through pure concepts of space, and the science that they determine, namely geometry, would not itself be possible" (B206-A166-B207). These remarks seem to indicate five things: 1) figures are possible as space that is enclosed, 2) geometry enables us to cognize a priori an enclosed space as space through intuition, 3) appearance is apprehended in a figure - a space that is enclosed: 4) all cognition of its objects is attained through the essential form of all intuition - the synthesis of spaces and times, 5) the synthesis of spaces and times is commensurate with the pure use of mathematics. What does space that is enclosed mean? It means space itself which is filled. Kant, first, suggests that a space which is enclosed is space itself. It is Kant who cognizes objects of the senses through empirical intuition, and comprehends them not as objects in themselves, but as mere appearances. This is wrong. The objects of the senses, which signify filled space-elapsing time, are the objects in themselves. However, as mentioned above, Kant, in an attempt to rectify this erroneous comprehension, has already inserted the phrase: "the synthesis of spaces and times, as the essential form of all intuition,..." (B206). Kant attempts to use "synthesis" as a devise to abstract "from everything empirical in the appearances" (A96), seemingly enabling him to apprehend appearances through "the essential form of all intuition." What does "the essential form of all intuition" mean? Emerging through the abstraction from everything empirical by means of mathematics, Kant's "synthesis of spaces and times, as the essential form of all intuition" appears to be correspondent to our "cognizing space-time itself through 
empirical intuition and synthesis.' However, they are entirely different on account of the fact that the former rests on the pure use of mathematics while the latter rests on sensibility.

For the time being, following our hypothesis, we attempt to find what empirical intuition meant among the standing and lasting Is, in a time prior to the advent of geometry or mathematics as a science - in primordial times. Then, we imagine without difficulty, that when the standing and lasting I happened to disappear in primordial times, this disappearance caused a starvation among the herd on account of the presumed fact that the herd lost this standing and lasting I, which could have been a hunter-gatherer. It is not farfetched to surmise that in primordial times, the loss of the person hunting for food had promptly caused starvation on the part of survivors in the herd. The loss of a feeder, which accounts for starvation, is to be cognized, through pure intuition affected by the sensation of lack of food on the part of survivors in the herd, as starvation, which is approximately homogeneous with death itself - nullity in space-time. We think that 1) a "part" of time itself, given as "enclosed between boundaries" (B211), i.e., between the presence of a certain amount of food and its absolute lack, is cognized to be a time - elapsing time - through empirical intuition; and 2) the absolute lack of food is cognized to be equivalent to nullified time through pure intuition affected by the sensation of lack of food - empirical intuition. Disappearance of the standing and lasting I occurs, as the dismemberment of itself. When the disappearance of another standing and lasting I occurs as its dismemberment, it follows that the standing and lasting I itself is to disappear in nullity in space-time, in experience or in possible experience, of starvation. We have to note that the empirical intuition in regard to starvation is homogeneous with pure intuition affected by the sensation of nullity empirical intuition in regard to death itself, since absolute lack of food - starvation - is tantamount to death itself in primordial times. We say that both 'elapsing time' and 'nullified time' are perceived through one and the same empirical intuition. Is it so? Yes, it is. Since the former is cognized through empirical intuition while the latter is cognized through pure intuition affected by the sensation of nullity - empirical intuition, there is no difference between the two. Since Kant thinks of neither axioms nor intuitions in regard to the existence of the representation 'I think,' he has to say that "if a perception is given to us in a temporal relation to others (even though indeterminate), it cannot be said a priori which and how great this other perception is, but only how it is necessarily combined with the first, as regards its existence, in the modus of time" (B222). Thinking that the empirical intuition in regard to starvation is homogeneous with empirical intuition in regard to death itself in primordial times, we say, in an opposite manner, that 'when a perception of nullity is given to us in a temporal relation to others (even though indeterminate), it can be said a priori, which, and how great, this other perception of nullity is, and how it is necessarily combined with the first perception of elapsing, as regards its existence, in the modus of time itself.' This signifies that the perception of nullified time, which is given to one in a temporal relation to others, is to be commensurate with the perception of nullified time in oneself which is to be fulfilled in possible experience. We say that others' deaths are as equivalent to the dissolution of filled space-elapsing time as one's death in possible experience is, which is to be perceived as nullity in space-time through empirical intuition and synthesis. Why did Kant miss the mark? It is because Kant thinks that time is not perceived through the modus of 'elapsing' or 'nullified,' but only through the modus of 'elapsing.' Contrarily, we think that since time itself - nullity in time - manifests itself in the modus of 'elapsing' or 'nullified' in the world of sense, time itself which is not the modus of time is to be perceived in the modus of nullity.

A serious problem concerning "the absolute magnitude of the series in this world" inevitably arises in Kant's metaphysics, which forces him to think that "the only thing left to us is the validity of the principle of reason as a rule for the continuation and magnitude of a possible experience, once its invalidity as a constitutive principle of appearances in themselves has been adequately demonstrated" (A516/B544). We think that Kant has not adequately demonstrated it. In regard to this issue, what Kant refers to gives us a hint which would lead us to the solution of Kant's conundrum. Accordingly, Kant says, "All appearances whatsoever are accordingly continuous magnitudes, either in their intuition, as extensive magnitudes, or in their mere perception (sensation and thus reality), as intensive ones. If the synthesis of the manifold of appearances is interrupted, then it is an aggregate of many appearances, and not really appearance as a quantum, which is not generated through the mere continuation of productive synthesis of a certain kind, but through the repetition of an ever-ceasing synthesis" (B212). This remark seems to indicate that 1) all appearances themselves which have continuous magnitudes, pertain to empirical intuition and signify reality, 2) appearance as a quantum is generated through the mere continuation of productive synthesis of a certain kind, 3) an aggregate of many appearances is not a quantum since it is generated through the repetition of an ever-ceasing synthesis, 4) magnitudes are to be made into a quantum by means of mere continuation of productive 
synthesis of a certain kind - a devise with which one "abstracts from everything empirical in the appearances" (A96). It seems that Kant thinks that since magnitude is the stumbling block for "the repetition of an ever-ceasing synthesis" of the manifold of appearances, it must be neutralized so as to be a quantum. In contrast, we think that 1) since all appearances themselves, which signify 'filled space-elapsing time' or 'empty space-nullified time,' are homogeneous with space-time itself, they are quantum and have continuous magnitude, 2) therefore, an aggregate of many appearances themselves, which is already quantum, is generated "through the repetition of an ever-ceasing synthesis" of the manifold of appearances which is not interrupted. Since it is apparent that the issue of appearances, magnitude and continuation are related to the issue of causality, Kant brings up the issue of causality of an alteration, saying that if the causality of an alteration in general is to be comprehended within the boundaries of empirical principles, that "all alteration (transition of a thing from one state into another) is also continuous" is easily proved by a transcendental philosophy (A171-B213). However he deplores that he cannot do it, saying that "the understanding gives us no inkling a priori that a cause is possible which alters the sate of thing, i.e., determines them to the opposite of a certain given state,...because alterability concerns only certain determinations of appearances, about which experience alone can teach us, while their cause is to be found in the unalterable" (B213). We have already shown, in our transcendental analytic that 1) the understanding gives us an inkling a priorideath - that a cause is possible which alters the state of thing: and 2) since alterability concerns only the determinations of appearances, namely filled space-elapsing time or empty space-nullified time, space-time itself is the unalterable - the cause. Therefore, we have to say, in an opposite manner to what Kant refers to (A171-B213), that 'since all appearances, considered extensively as well as intensively, are continuous magnitudes, the proposition that all alteration of a thing from one state into another is also continuous is proved easily and with mathematical-evidence...the understanding gives us an inkling $a$ priori that a cause is necessary and universal which alters the state of things, i.e., determines them to the opposite of a certain given state, not merely because it gives us insight into the necessity and the universality of this, but because alterability concerns certain determinations of appearances about which experience alone can teach us, while their cause is to be found in the unalterable.' Now, since one presupposition has been proved, we can enhance the empirical intuition from the "subjective validity, e.g., in accordance with laws of association" (B142), to the objective validity in transcendental deduction. What is this presupposition? The presupposition that "all appearances whatsoever are accordingly continuous magnitudes, either in their intuition, as extensive magnitudes, or in their mere perception (sensation and thus reality), as intensive ones" (B212) has been proven in such a way that continuous magnitude is to be cognized, through empirical intuition, that it signifies, as object in itself, nullity in space-time. We think that the fact that 'alterability concerns only the determinations of appearances, namely filled space-elapsing time or empty space-nullified time, space-time itself is the unalterable - the cause' indicates that "one reality, if combined in one subject with another, cancels out the effect of the latter, which is unceasingly placed before our eyes by all hindrances and countereffects in nature, which, since they rest on forces, must be called realitates phaenomena" (B329). Now since the synthesis of the manifold of appearances themselves is not interrupted, it becomes possible for us to attain the comprehension of "a completed synthesis and the consciousness of its absolute totality" through empirical cognition (A482/B510-B511-A483), opening a way to the "metaphysical deduction" in which "the origin of the a priori categories in general" is "established through their complete coincidence with the universal logical functions of thinking" (B159).

When we add synthesis to empirical intuition here, we partly agree with what Kant refers to in regard to category and the understanding, and partly disagree. As for category Kant says, "Categories are concepts that prescribe laws a priori to appearances, thus to nature as the sum total of all appearances (natura materialiter spectata), and, since they are not derived from nature and do not follow it as their pattern..." (B163). Contrary to Kant, we say that 1) nature prescribes laws a priori to appearance, 2) the law of nature, as the sum total of all appearances, prescribes concepts as categories, 3) thus, categories are concepts that prescribe laws a priori to appearances. As for the understanding, Kant says, "our understanding, according to which it is only through the perception and comparison of sequences of many occurrences on preceding appearances that we are led to discover a rule, in accordance with which certain occurrences always follow certain appearances, and are thereby first prompted to form the concept of cause. On such a footing this concept would be merely empirical, and the rule that it supplies, that everything that happens has a cause, would be just as contingent as the experience itself: its universality and necessity would then be merely feigned, and would have no true universal validity, since they would not be grounded a priori but only on induction" (A195-B241-A196). Contrary to Kant, we say that "the concept of cause, which has a footing on the law of nature, would be empirical, and the law that it supplies, that everything that happens has a 
cause, would never be contingent in a like manner as death itself: its necessity and universality would have true universal validity since they would be grounded a priori on experience or possible experience, and on deduction as well.' Furthermore, following what Kant refers to concerning apprehension and perception (A196-B242), we say that we have shown an example that, in experience and in possible experience, we ascribe sequences of an occurrence in which something happens that previously existed, to death itself - object in itself - which belongs to the category of the pure concepts of the understanding (YAMAMOTO 2016: 87-100). Therefore, we do not distinguish the sequence of an occurrence from the subjective sequence of our apprehension, since the law of nature "is the ground that necessitates us to observe this order of the perceptions rather than another, indeed that it is really this necessitation that first makes possible the representation of a succession in the object" (A196-B242-A197). In regard to the issue of apprehension, Kant says, "in experience, to be sure, perceptions come together only contingently, so that no necessity of their connection is or can become evident in the perceptions themselves, since apprehension is only a juxtaposition of the manifold of empirical intuition, but no representation of the necessity of the combined existence of the appearances that it juxtaposes in space and time is to be encountered in it" (B219). No, this is wrong. Contrary to Kant, we have to say that in experience and in possible experience, the "inner perception of the manifold that is antecedently given in the subject" (B68) comes together necessarily, so that the "necessity of their connection is or can become evident in the perceptions themselves," since apprehension is "a juxtaposition of the manifold of empirical intuition," and a "representation of the necessity of the combined existence of the appearances that it juxtaposes in space and time is to be encountered in it." We would say so, on account of the presumed fact that the decease of one of the standing and lasting Is would invariably precipitate apprehension among others in the herd when the manifold of empirical intuition, namely nullity in space-time, is juxtaposed. It would become explicit in the juxtaposition of disappearance that a human, as appearance, is in the combined existence of the appearances. Furthermore, following what Kant refers to (B218-B219), we say in an opposite manner, that 'since time itself can be perceived as nullity in time, the determination of the existence of objects in time can come about through their combination in time, hence, through a priori connecting concept, i.e., nullity in time. It is therefore a synthesis of perceptions, which is itself contained in perception, and contains the empirical unity of the manifold of perception in one consciousness which constitutes what is essential in a cognition of objects themselves.' We also say that the cognition of objects themselves is to be attained through empirical intuition, in experience and possible experience.

Kant elaborates on the synthesis of perceptions, saying "...all combinations, whether we are conscious of it or not, whether it is a combination of the manifold of intuition or of several concepts, and in the first case either of sensible or non-sensible intuition, is an action of the understanding, which we would designate with the general title synthesis in order at the same time to draw attention to the fact that we can represent nothing as combined in the object without having previously combined it ourselves, and that among all representations combination is the only one that is not given through object but can be executed only by the subject itself, since it is an act of its self-activity" (B130). Here, it is clear that Kant needs something that invokes him to take the act of self-activity synthesis. It is very unfortunate for Kant that he has nothing but "an act of the spontaneity of the power of representation" - understanding (B130). We agree with Kant, who indicates that humans should have taken the "act of the spontaneity of the power of representation" from primordial times, since it is clear that synthesis - "an action of the understanding" (B130) is indispensable for combining the manifold of appearance (filled space-elapsing time) with the manifold of disappearance (empty space-nullified time), thereby comprehending the phaenomena of appearing and its disappearing in regard to the standing and lasting I to be the law of nature. Our understanding concerning the phaenomena of appearing and its disappearing is "only through the perception and comparison of sequences of many occurrences on preceding appearances" (A195). Since Kant says "the schema of necessity is the existence of an object at all times" (A145), we have to search for the schema of necessity through empirical intuition and synthesis in order to cognize the existence of an object in itself. Examining the scheme of empirical intuition and synthesis, whose elements, we think, reside in the manifold of intuition, namely in filled space-elapsing time, and vanish in empty space-nullified time, we come across the fact that space-time itself and the form of appearances in terms of 'filled-elapsing' or 'empty-nullified' is the necessity at all times, indicating that these are, as the schema of necessity, equivalent to the existence of an object in itself. Since space-time itself always inheres in the standing and lasting I, of which empirical intuition and synthesis are constituents, we think that space-time itself - nullity in space-time - inheres in empirical intuition and synthesis. In other words, the standing and lasting I of pure apperception exists in nullity in space-time, while it appears in filled space-elapsing time. Upon dismemberment of the manifold of 
intuition, filled space-elapsing time is neutralized, and it would vanish in nullity in space-time, returning to space-time itself. In regard to the issues of appearances and things in themselves, Kant explicitly says "if appearances were things in themselves, and so just for this reason their condition always belong to one and the same series of intuitions, then a necessary being could never occur as a condition of the existence of appearances in the world of sense" (A559/B587-A560/B588). We say, in an opposite manner, that 'appearances themselves are things in themselves - filled space-elapsing time or empty space-nullified time - and so just for this reason their condition always belongs to one and the same series of intuitions - space-time itself, therefore a necessary being could occur as a condition of the existence of appearances in the world of sense.' What does this mean? It means that appearance itself - filled space-elapsing time - appears distinct from a thing in itself - nullity in space-time. Since, space-time itself appears in the mode of 'filled-elapsing' or 'empty-nullified,' all appearances - appearance and its disappearance - do not appear to belong to one and the same series of intuition, though they belong to one and the same series of intuition. We think this is the origin of Kant's missing of the mark. Contrary to Kant, we say that if a necessary being could occur as a condition of the existence of appearances in the world of sense, it is nothing but space-time itself and the form of appearances in terms of 'filled-elapsing' or 'empty-nullified.' The crucial point here is that an appearance contingently appears in filled space-elapsing time, while it necessarily disappears in nullity in space-time. A necessary being is to be comprehended by means of the understanding that disappearance - death itself - signifies nullity in space-time, through empirical intuition and synthesis. Here we have to take note that 'empirical intuition' does not necessarily correspond to 'empirical intuition and synthesis,' on account of the fact that synthesis signifies "an act of the spontaneity of the power of representation," "in distinction from sensibility" (B130). We think that this act of the spontaneity of the power of representation, in distinction from sensibility, signifies the origin of humanity itself.

The origin of the critical issue of magnitude, which emerges as the antinomy of pure reason in the system of cosmological ideas, is to be found in Kant's own words, which says "All appearances contain, as regards their form, an intuition in space and time, which grounds all of them a priori. They cannot be apprehended, therefore, i.e., taken up into empirical consciousness, except through the synthesis of the manifold through which the representations of a determinate space and time are generated, i.e., through the composition of that which is homogeneous and the consciousness of the synthetic unity of this manifold (of the homogeneous). Now the consciousness of the homogeneous manifold in intuition in general, insofar as through it the representation of an object first becomes possible, is the concept of a magnitude (Quanti). Thus even the perception of an object, as appearance, is possible only through the same synthetic unity of the manifold of given sensible intuition through which the unity of the composition of the homogeneous manifold is thought in the concept of a magnitude, i.e., the appearances are all magnitudes, and indeed extensive magnitudes, since as intuitions in space or time they must be represented through the same synthesis as that through which space and time in general are determined. I call an extensive magnitude that in which the representation of the parts makes possible the representation of the whole (and therefore necessarily precedes the latter)" (B202-B203-A162). Here, almost everything concerning the origin of the conundrum of Kant's metaphysics in terms of magnitudes and his scheme of an attempt to solve it can be seen. Why cannot Kant reach the shore of things in themselves? First, Kant's view that "all appearances contain, as regards their form, an intuition in space and time" is absolutely wrong, since we think that all appearances themselves contain, as regards their condition, "an intuition in space and time, which grounds all of them a priori." Second, Kant's view that "They cannot be apprehended,...except through the synthesis of the manifold" is wrong. We think that the condition, which all appearances themselves contain, can be apprehended through empirical intuition and resultant synthesis. Third, Kant's view that "the perception of an object, as appearance, is possible only through the same synthetic unity of the manifold of given sensible intuition through which the unity of the composition of the homogeneous manifold is thought in the concept of a magnitude" is wrong. We think that perception of an object in itself, as appearance itself (i.e., filled space-elapsing time or empty space-nullified time) is possible, only as a possible experience, through another empirical unity of the manifold of given sensible intuition (i.e., filled space-elapsing time) through which the unity of the composition of the homogeneous manifold (i.e., space-time itself) is thought of in the concept of a magnitude. Fourth, Kant's view that "I call an extensive magnitude that in which the representation of the parts makes possible the representation of the whole (and therefore necessarily precedes the latter)" is wrong. We think that though an extensive magnitude is "that in which the representation of the parts makes possible the representation of the whole," it does not necessarily precede the latter on account of the fact that magnitude in the parts and the whole is already Quanti. At the same time, this is one of the most important confessions on the part of Kant, 
since it exposes Kant's deliberate design in the Critique of Pure Reason. What is it? His opaque design is this: To alienate empirical intuition from which magnitudes are thought to be derived, from synthesis - "an action of the understanding" (B130), through which magnitudes are to be nullified, thus eliminating magnitudes from appearances in the world of sense by means of Kant's pure reason. Kant's metaphysics says, "No one can define the concept of magnitude in general except by something like this: That it is the determination of a thing through which it can be thought how many units are posited in it. Only this how-many-times is grounded on successive repetition, thus on time and the synthesis (of the homogeneous) in it. Reality, in contrast to negation, can be defined only if one thinks of a time (as the sum total of all being) that is either filled by it or empty" (B300). We partly agree and partly disagree with Kant, thinking that 1) a human is to perceive time itself in negation through empirical intuition and synthesis, when magnitude, as the homogeneous in sensation, comes into view as a result of a possible experience of death - the absolute cessation of successive repetition, and to perceive magnitude in reality when nullity in time comes into view as a result of a possible experience of death - absolute cessation of successive repetition: 2) it is death itself that exposes magnitude, reality and time itself: 3) "reality, in contrast to negation," emerges when space itself is 'filled' and time itself is 'elapsing,' while reality in conformity with negation emerges when a space is made 'empty' and a time is made 'nullified:' 4) "how many units are posited in" appearance itself can be its determination since the units signify the homogeneous as empty space-nullified time or filled space-elapsing time - appearance itself. When Kant cannot solve the conundrum in regard to magnitudes though mere understanding, he can do nothing but resort to pure reason in order to solve it. Kant explicates the direction of his discourse, saying, "if one does away with all conditions of sensibility that distinguish them as concepts of a possible empirical use, and takes them for concepts of things in general (thus of transcendental use), then that is to do nothing more than to regard the logical functions of judgments as condition of the possibility of things themselves, without in the least being able to show whence they could have their application and their objects, thus how in pure understanding without sensibility they could have any significance and objective validity" (A241-A242-B300). Though "the logical functions of judgments" are expected to play the role of condition of the possibility of things in themselves here, Kant cannot achieve his aim because he fails to comprehend ontology, i.e., the schema of "a priori cognitions of things in general in a systematic doctrine (e.g., the principle of causality)" (A247), with transcendental analytic. The only one that is possible for Kant is "pure understanding without sensibility" or "a pure category, in which abstraction is made from any condition of sensible intuition" (B304). We think that Kant's failure to achieve his aim forced him to think of things in themselves, namely pure category, which "does not suffice any synthetic a priori principle" (A248). He has to think of it by means of his 'pure reason' since things in themselves and magnitudes can be dialectic under the aegis of his pure reason. However, in fact, Kant has already suggested that "all magnitudes" in regard to the "appearances" are considered to be "the homogeneous manifold" (B203) - quantum. Kant seems to have already solved his whole conundrum. Nevertheless, the conundrum in Kant's metaphysics issues from his contradictory way of thinking in regard to magnitudes, which suggests that if an object, as appearance, is "taken up into empirical consciousness" through empirical intuition, it is apprehended in intensive magnitudes, while, if it is apprehended "through the synthesis of the manifold" as the representation of an object with the same empirical unity, it is comprehended as Quanti (B202-B203). We have to note that a human perceives, through empirical intuition and synthesis, nullity in the possible milieu of the sensation of nullity in space-time, or through empirical intuition, nullity in the milieu of the sensation of nullity in space-time. This indicates that perception is attained, through one and the same empirical intuition, differently, on account of the fact that death comes across humans as a thing in itself or as a possibility. However, the fact that this 'possibility' is under the aegis of the law of nature implies that it is to be equivalent to thing in itself. Does not Kant take this difference between 'nullity in the possible milieu of the sensation of nullity in space-time' and 'nullity in the milieu of the sensation of nullity in space-time' as the difference between appearance and thing in itself? We say, 'Yes, he does.' Why? Mathematics as a science leads him to abstract from the milieus of the sensation of nullity in space-time, congealing it into "a necessity of synthetic unity" (B234) - "a merely intelligible object," "about which one knows nothing" (A565/B593).

Kant differentiates empirical intuition from empirical intuition and synthesis by adding synthesis to empirical intuition, concomitantly making magnitudes into a quantum. In contrast to Kant, we think that the perception of an object in itself, i.e., nullity in space-time through empirical intuition, corresponds to that through empirical intuition and synthesis. In this regard, the issue of magnitudes concerning time is neutralized with little difficulty. Referring to the schematism of the pure concepts of the understanding, Kant says "the schema of each category contains and makes representable: in 
the case of magnitude, the generation (synthesis) of time itself, in the successive apprehension of an object;..." (A145). This enigmatic remark seems to indicate that 1) magnitude is generated in the synthesis of time itself, 2) time is generated in the successive apprehension of an object in itself, 3) time and magnitude become representable through "the schema of each category" which contains both. We think that since magnitude and time itself are quantum, "the schema of each category" is quantum. Therefore, time which is generated in the successive apprehension of an object in itself is homogeneous with the magnitude which is generated in the synthesis of time itself, indicating that an object in itself is a quantum. Since magnitude, time itself and object in itself as categories signify quantum, these categories, as "the representation of appearance; that the things that we intuit," are "in themselves what we intuit them to be," and "are their relations so constituted in themselves as they appear to us" (A42). This metaphysics will open the way through which it is possible to reach "the possibility of a world-whole itself," provided the ground of explanation would be within the world of sense and hence it would be "an object of a possible experience" (A677/B705). We think that the successive apprehension of an object in itself takes place in the successive encounters, in apprehension, with death itself, on the part of a subject, either in experience or in possible experience. Death itself - as "the schema of necessity" (A145) under the aegis of the law of nature - signifies the existence of an object in itself at all times in virtue of nullity in space-time (YAMAMOTO 2016: 87-100). Thus, we say that an object in itself corresponds to death itself, whose synthesis occurs as a result of apprehension of death itself - nullity in space-time. Here, it is not farfetched to suppose that magnitude in regard to time is commensurate with nullity in time. In this context, "an absolute totality of conditions" (B384) in regard to time become comprehensible in such a way that the absolute totality is already "intuited as aggregates (multitudes of antecedently given parts)" (B204) in nullified time. Thus, we think that the "multitudes of antecedently given parts" - quantum - is already equal to the aggregates of them - quantum. Since magnitude in time, which is commensurate with nullity in time, is represented and apprehended as continuous, it can be said that intensive magnitude in time signifies 'elapsing time.' We say that 'since intensive magnitude of contingent things appears in elapsing time, it appears to be sensible as "quantum discretum" (A526/B554), and is sensible as such, while since extensive magnitude of things in themselves is nullity in time, it is cognized as "quantum continuum" (A527/B555) when it emerges upon the entire dissolution of contingent things.' In relation to this issue, Kant furthers the issue of magnitudes as follows: "The pure schema of magnitude (quantitatis), however, as a concept of the understanding, is number, which is a representation that summarizes the successive addition of one (homogenous) unit to another. Thus number is nothing other than the unity of the synthesis of the manifold of a homogeneous intuition in general, because I generate time itself in the apprehension of the intuition" (B182-A143). Contradictorily, Kant, as if he forgets this remark, says, "The infinite division indicates only the appearance as quantum continuum, and is inseparable from the filling of space; for the ground of its infinite divisibility lies precisely in that. But as soon as something is assumed as a quantum discretum, the multiplicity of units in it is determined; hence it is always equal to a number" (A527/B555). Is the former number - "a concept of the understanding" - which is supposed to be equivalent to "the pure schema of magnitude (quantitatis)" different from the latter, which is supposed to be equivalent to "the multiplicity of units" in something assumed as a quantum discretum? No, they are not different, but the same. The former number - "a representation that summarizes the successive addition of one (homogenous) unit to another" - signifies quantum continuum in terms of nullity in space-time, while the latter number, which signifies quantum continuum, appears to be quantum discretum under the assumption that a thing signifies quantum discretum. In Kant's metaphysics, number - quantum continuum - is homogeneous with "the appearance as quantum continuum." However, when it is under the aegis of Kant's assumption, number is transformed into one in which the multiplicity of units is determined. This metaphysics appears to be in contradiction with our empirical intuition and understanding. Instead of Kant, we have to say 'a number - quantum continuum - is homogeneous with the appearance itself, and appears to be homogeneous with the multiplicity, insofar as thing in itself - space-time itself - appears as quantum discretum.' We comprehend, through empirical intuition and synthesis - "the apprehension of the intuition" (A143) - that upon the entire cessation of movement of the standing and lasting I, number and magnitude emerges as quantum continuum, while during its movement, number and magnitude appear as quantum discretum. Therefore, it can be said that number and magnitude enables the standing and lasting I of pure apperception to generate time itself in quantum continuum and determines 'elapsing time' as quantum discretum. In other words, time itself - "the absolute whole of magnitude (the world-whole)" (A483) - emerges from number and magnitude as quantum continuum which is homogeneous with number and magnitude of 'the standing and lasting Is,' which existed or exist or will exist while a time - "every determinate magnitude of time" (A32-B48) - emerges from number and magnitude as quantum discretum, which 
is homogeneous with number and magnitude of the standing and lasting Is, which exist. In view of this, our discourse can be said to agree with Kant's metaphysics, which says, "Every intuition contains a manifold in itself, which however would not be represented as such if the mind did not distinguish the time in the succession of impressions on one another; for as contained in one moment no representation can ever be anything other than absolute unity" (A99). Our metaphysics, in conformity with Kant's, says that 1) one moment in which appearance disappears signifies the representation of "a manifold in itself," which is equivalent to "absolute unity" - nullity in time: 2) intuition is a manifold in itself - absolute unity - insofar as the mind distinguishes nullity in time in one moment "in the succession of impressions on one another." What does "the succession of impressions on one another" mean? We have already explicated it above, saying, 'Disappearance of the standing and lasting I occurs, as the dismemberment of itself. When the disappearance of another standing and lasting I occurs as its dismemberment, it follows that the standing and lasting I itself is to disappear in nullity in space-time, in experience or in possible experience, of starvation. We have to note that the empirical intuition in regard to starvation is homogeneous with pure intuition affected by the sensation of nullity - empirical intuition in regard to death itself, since absolute lack of food starvation - is tantamount to death itself in primordial times.' Therefore, we say that 1) appearances in the world of sense, including the standing and lasting I are cognized empirically as filled space-elapsing time: 2) nullity in space-time always inheres in the standing and lasting I which appears in the world of sense. In contrast to what Kant refers to concerning transcendental logic (A72), we say that our transcendental analytic "considers the value or content of the logical affirmation made in a judgment by means of" a negative predicate and the "gain this yields for the whole of cognition." Furthermore, following what Kant refers to (B202-B203), we assert that 'All appearances contain, in regard to their condition, an intuition - nullity in space-time - which grounds all of them a priori. They can be apprehended, i.e., taken up into empirical consciousness, through empirical intuition and the synthesis of the manifold, through which the representations of a determinate space and time space-time itself - are generated as nullity, i.e., through the composition of nullity which is homogeneous and the consciousness of the empirical unity of this manifold of the homogeneous.'

Here, we have to turn to the issue of magnitudes in space. In regard to space, Kant says, "The pure image of all magnitudes (quantorum) for outer sense is space" (B182). Kant clarifies how humans cognize space itself upon encountering the manifold of appearance itself, saying, "as contained in one moment no representation can ever be anything other than absolute unity. Now in order for unity of intuition to come from this manifold (as, say, in the representation of space), it is necessary first to run through and then to take together this manifoldness, which action I call the synthesis of apprehension, since it is aimed directly at the intuition, which to be sure provides a manifold but can never effect this as such, and indeed as contained in one representation, without the occurrence of such a synthesis" (A99). We think that this enigmatic remark indicates five things: 1) humans intuit space itself in 'running through' the manifold of appearance: 2) 'running through' is the representation of space itself, which is taken together, spawning the unity of intuition: 3 ) this manifoldness (running-through) is the negative effect on sensibility, which is commensurate with pure intuition affected by the sensation of nullity - empirical intuition: 4) taking together the 'running-through' through empirical intuition is the synthesis of apprehension; and 5) 'running-through' is one representation - "unity of intuition" which is homogeneous with "absolute unity," i.e., nullity in space. We agree with Kant, thinking that this is the "pure image of all magnitudes (quantorum) for outer sense" (B182), i.e., space itself, provided that 'running-through' the manifold of appearance and the synthesis of apprehension are thought to take place when a human, as a manifold of appearance, encounters the manifold of its disappearance which occurs necessarily and universally among the manifold of appearance. In regard to the unity of intuition and the manifold of disappearance, Kant also makes a suggestive remark, saying, "The synthesis of apprehension is therefore inseparably combined with the synthesis of reproduction. And since the former constitutes the transcendental ground of the possibility of all cognition in general (not only of empirical cognition, but also of pure a priori cognition), the reproductive synthesis of the imagination belongs among the transcendental actions of the mind, and with respect to this we will also call this faculty the transcendental faculty of the imagination" (A102). We think that this remark indicates that 1) both empirical cognition and "pure a priori cognition" rest on the "synthesis of apprehension," 2) the synthesis of apprehension occurs in conjunction with the "synthesis of reproduction," 3) the synthesis of reproduction rests on the "transcendental actions of the mind" - the "transcendental faculty of the imagination." We think that pure a priori cognition, which Kant thinks is in distinction from empirical cognition, is meant to correspond to cognizing nullity through pure intuition affected by the sensation of nullity - empirical intuition. It rests on the transcendental actions of the mind, since cognizing nullity empirically is equivalent to cognizing 
phaenomena of disappearance of the manifold of intuition through empirical intuition and synthesis of apprehension. A standing and lasting I and another standing and lasting I come to be able to cognize object in itself when the mind is acted on by the sensation of nullity in space-time - death itself - in experience or in possible experience. The transcendental actions of the mind, which are spontaneous on account of the fact that it is acted on by the sensation of nullity, causes the "reproductive synthesis of the imagination" as a result of apprehension. Since apprehension arises in conjunction with anxiety, dread, fear or astonishment, the synthesis of apprehension is not separated from the sensation of nullity, which is aroused upon encountering death itself in possible experience. Thus, "a manifold in itself" - death itself - can be thought to signify nullity in space-time, which is to be cognized in experience or in possible experience of death itself. Within the same view, we say that the thinking subject as a representation of the manifold in itself signifies something positive in space-time (as filled space-elapsing time). At the same time, this subject 'as the condition of the manifold of inner sense, thus of the connection of all representations, contains an a priori manifold, namely death, in pure intuition as nullity' (YAMAMOTO 2016: 87-100). In this context, it becomes comprehensible that "the absolute unity of the thinking subject" (A335) is homologous with nullity in space-time.

In regard to the issues of the manifold of appearance and the manifold in itself, Kant makes a suggesting remark, saying, "Our apprehension of the manifold of appearance is always successive, and is therefore always changing. We can therefore never determine from this alone whether this manifold, as object of experience, is simultaneous or successive, if something does not ground it which always exists, i.e., something lasting and persisting, of which all change and simultaneity are nothing but so many ways (modi of time) in which that which persists exists" (A182-B226). We agree with Kant and say, 'our apprehension of the manifold of appearance (e.g., a human) is successive but can never be always successive since successive appearance of the manifold is to be interrupted on account of its disappearance, which is necessary and universal. We can therefore determine from this whether this manifold, as object of experience, is simultaneous or successive.' In our metaphysics, "something lasting and persisting, of which all change and simultaneity are nothing but so many ways (modi of time) in which that which persists exists" (A182-B226), is space-time itself - nullity in space-time. Therefore, cognizing the manifold of appearance in filled space-elapsing time, which is the object of experience, and the manifold of disappearance in empty space-nullified time, which is object of possible experience, we determines whether the manifold, as an object of experience or an object of possible experience, is simultaneous or successive on the ground of something lasting and persisting - space-time itself. Furthermore, in association with this issue, Kant makes important remarks, saying, "I call that in the appearance which corresponds to sensation its matter, but that which allows the manifold of appearance to be intuited as ordered in certain relations I call the form of appearance. Since that within which the sensation can alone be ordered and placed in a certain form cannot itself be in turn sensation, the matter of all appearance is only given to us a posteriori, but its form must all lie ready for it in the mind a priori, and can therefore be considered separately from all sensation" (A20). We think that space-time itself - nullity in space-time - is the matter, namely "that in the appearance which corresponds to sensation," and that 'filled-elapsing' or 'empty-nullified' is the form of appearance "which allows the manifold of appearance to be intuited as ordered in certain relations." Therefore, we say, in an opposite manner, "since matter - "that in the appearance which corresponds to sensation" - within which the sensation can be ordered and placed in a certain form can be in turn sensation, the matter of all appearance is given to us a priori, and its form must all lie ready for it in the mind a priori, and cannot therefore be considered separately from all sensation.' We think that 1) "a whole existing in itself" is equivalent to the world - "the sum total of all appearances (B535-A507): and 2) Kant's "all appearances" signifies appearance and disappearance, namely "manifold, as object of experience," and "a priori manifold" (A138/B177) which are homogeneous with "manifold in itself" (A99). The world emerges as "the sum total of all appearances" - the sum total of manifold in itself - which is the "world-whole itself" (A677/B705). Since the "world-whole itself" should be commensurate with "a whole existing in itself," it is homogeneous with the whole "substance" (B321) - filled space-elapsing time and empty space-nullified time. Therefore, we say that "every substance" - substantia phaenomenon - has "inner determinations and forces that pertain to its inner reality" (B321). In view of our discourse that the matter is space-time itself and the form is 'filled-elapsing' or 'empty-nullified,' we say that Kant's "all appearances whatsoever are accordingly continuous magnitudes" (B212) or objects which "are given to us in intuition" (B87) signify filled space-elapsing time or empty space-nullified time. Furthermore, we say, following what Kant says (A42), 'All our intuition is nothing but the representation of all appearances.' Indeed, "Every intuition contains a manifold in itself' (A99). When Kant, following the aforementioned remarks (A20), hastily adds, saying, "I call all representations pure (in the transcendental sense) in which nothing is to be encountered that belongs to sensation. Accordingly the pure form of sensible intuitions in general is to 
be encountered in the mind a priori, wherein all of the manifold of appearances is intuited in certain relations. This pure form of sensibility itself is also called pure intuition. So if I separate from the representation of a body that which the understanding thinks about it, such as substance, force, divisibility, etc., as well as that which belongs to sensation, as impenetrability, hardness, color, etc., something from this empirical intuition is still left for me, namely extension and form. These belong to the pure intuition, which occurs a priori, even without an actual object of the senses or sensation, as a mere form of sensibility in the mind" (A20-B35-A21), we say that this is a tricky remark. Thinking that there is a representation, which appears pure (in the empirical sense) in which nullity is to be encountered that pertains to sensation, we say that Kant tries to neutralize this way of thinking by means of "the transcendental sense," abstracting sensation from all representations in which 'a representation, which appears pure (in the empirical sense), is comprised. Then, something left from this abstraction is the "pure form of sensibility itself," namely "extension and form." They are thought to belong to "pure intuition, which occurs a priori," which is homogeneous with "an a priori manifold in pure intuition" (A138/B177). Since a priori manifold in pure intuition signifies nullity (YAMAMOTO 2016: 87-100), pure intuition is homogeneous with nullity. However, this nullity in the mind is grounded as "a mere form of sensibility" on the act of abstraction on the part of Kant, while our nullity in space-time is grounded as "the representation of a body" on empirical intuition and resultant synthesis. In Kant's metaphysics, "extension and form" comes out as nullity itself, which is thought to belong to the pure intuition, which occurs as "a mere form of sensibility in the mind" (A21). Yes, pure intuition "occurs a priori even without an actual object of the senses or sensation." However, it is not "a mere form of sensibility in the mind," but a necessary condition of sensibility in the mind. We have to say that pure intuition is a priori insofar as it is not a mere form, but a necessary condition. As a necessary condition, pure intuition pertains to space-time itself. Kant, in separating substance, force, divisibility or impenetrability, hardness, color, etc., from the representation of a body, attempts to abstract from empirical intuition, which is expected to make "all representations pure (in the transcendental sense) in which nothing is to be encountered that belongs to sensation" (A20). What is left from this abstraction is "extension and form" - a figment of the imagination. Thus, Kant obliterates body in his metaphysics. This is wrong. We have to think that "extension and form" are to pertain to substance, force, divisibility, etc., or to impenetrability, hardness, color, etc., since it is a thing in itself existing in space-time itself, but appears in filled space-elapsing time. Though Kant discusses almost nothing about disappearance in the Critique of Pure Reason, when he makes remarks such as this: "how a subject can internally intuit itself; yet this difficulty is common to every theory. Consciousness of itself (apperception) is the simple representation of the I, and if all of the manifold in the subject were given self-actively through that alone, then the inner intuition would be intellectual. In human beings this consciousness requires inner perception of the manifold that is antecedently given in the subject, and the manner in which this is given in the mind without spontaneity must be called sensibility on account of this difference" (B68), we feel that Kant has already discussed, unwittingly, on the role of which disappearance plays in metaphysics. We adds, saying, in an opposite manner to what Kant says (B68-B69), 'If the faculty of becoming conscious of oneself is to seek out (apprehend) that which lies in the mind, it must not affect the latter, and it cannot produce an intuition of itself in such a way, whose condition, which antecedently grounds it in the mind, determines the way in which the manifold is together in the mind in the representation of time.' We think that the alteration of the form of appearances from 'filled-elapsing' to 'empty-nullified' affects the mind, determining an intuition and time in such a "way in which the manifold is together in the mind in the representation of time" (B68-B69). While time itself - nullity in time - is homogeneous with "a pure inner intuition" (A124), the representation of time - elapsing time or nullified time - is also with a pure inner intuition. Since "time is certainly something real" (A37), elapsing time or nullified time is also something real. Humans are to encounter this something real in experience or in possible experience. Therefore, we can say, "I really have the representation of time and my determinations in it" (A37-B54). The representation of time is to be regarded really as object in itself and "as the way of representing myself as object" (B54). We adds, saying, in an opposite manner to what Kant says (B54), 'if I or another being could intuit myself with this condition of sensibility, then these very determinations, which we now represent to ourselves as alterations, would yield us a cognition in which the representation of time, and thus also of alteration, would occur.' It is regretful that this metaphysics does not permeate in Kant's entire discourse.

Since we have already clarified that the "unity of the synthesis of the manifold, outside or within us, hence also a combination with which everything that is to be represented as determined in space or time must agree, is already given a priori, along with these intuitions, as condition of the synthesis of all apprehension" (B161), we agree with what Kant says (B160). Here, we had better say, "we have 
outer as well as inner sensible intuition a priori in the representation of space-time, and the synthesis of the apprehension of the manifold of appearance must always be in agreement with the latter,... space-time itself is represented a priori not merely as sensible intuition, but also as intuitions themselves (which pertain to a manifold), and thus with the determination of the unity of this manifold in them.' This saying indicates that 1 ) humans have sensibility a priori, which pertains to intuitions themselves and the representation of space-time: 2) the synthesis of apprehension must be in agreement with this sensibility: 3) space-time itself and the representation of space-time - filled space-elapsing time or empty space-nullified time - are "the determination of the unity" of the "manifold" in humans. This implies that nullity in space-time permeates, as the determination, among humans and the whole living things.

\section{Magnitude, Degree, Reality ANd Continuity}

So far, we have discussed that 1) disappearance of a human is cognized, through empirical intuition and synthesis, as nullity in space-time: 2) a human which appears as an object before our eyes is cognized as something in which nullity in space-time is made 'filled' in space and 'elapsing' in time: 3 ) therefore, all appearances themselves signify 'filled space-elapsing time' or 'empty space-nullified time:' 4) a thing which disappeared in nullity in space-time is perceived as quantum continuum while a thing which appeared as an object before our eyes is perceived as quantum discretum: 6) a thing in itself, which appears (B324) - space-time itself - signifies quantum continuum, but appears as quantum discretum; and 7) quantum continuum and quantum discretum are perceived through one and the same series of empirical intuitions. Here, we have to note that the sensation of nullity, which pertains to loss or deletion or decease, is homogeneous with that of nullity in space-time, while the sensation of impenetrability, hardness, color, etc. (B35-A21), which pertains to being, net, or gain, is homogeneous with that of appearance itself as filled space-elapsing time. Furthermore, substance, force, divisibility, etc. about which the understanding thinks as the representation of a body (B35-A21) also pertain to the manifold of intuition - appearance itself. The fact that the difference of the sensation or that of the representation of a body pertains to appearance itself, indicates that the sensation or the representation pertains to "substantia phaenomenon." Since only nullity in space-time does not pertain to the difference of sensation and representation in experience or in possible experience, it manifests itself to be matter - space-time itself - in "substantia phaenomenon" (B333). Furthermore, we have to emphasize, saying again, that 'a thing which appeared as an object before our eyes is perceived as quantum discretum, since a thing in itself - space-time itself - "appears" as quantum discretum. This perception does not come merely through empirical intuition, but through empirical intuition and synthesis - the anticipation of perception.' Space-time itself, in our discourse, seems to correspond to Kant's transcendental ideality of time and space. In order to understand this, we have to say, first, in an opposite manner to what Kant refers to in regard to time (B52-A36), that 'we do not dispute all claims of time to absolute reality, namely where it would attach to things absolutely as a condition or property, even without regard to the form of sensible intuition. Such properties, which pertain to things in themselves, can be given to us through the senses. In this, therefore, consists the transcendental ideality of time, according to which it is nullity in time, even if one does not abstract from the subjective conditions of sensible intuition, and can be counted as either subsisting or inhering in the objects in themselves.' In regards to space, we have to say, in an opposite manner to what Kant refers to (A28), 'we assert the empirical reality of space (with respect to all possible outer experience), and to be sure its transcendental ideality, i.e., that it is nullity in space if we do not leave aside the condition of the possibility of all appearance, and take it as something that grounds the things in themselves.' Furthermore, we have to say, in opposition to Kant (A30), that 'The transcendental concept of appearances in space is a critical reminder that 1) nullity that is intuited in space is a thing in itself: 2) space is a condition that is proper to anything in itself: 3) objects in themselves are known to us; and that 4) what we call outer objects are not mere representations of our sensibility, whose condition is space itself, and whose true correlate, i.e., the thing in itself, is, and can be, cognized through them, and is also sought after in experience or in possible experience.' Kant adamantly opposes this way of thinking, saying "The principle of continuity forbade any leap in the series of appearances (alterations) (in mundo non datur saltus), but also any gap or cleft between two appearances in the sum of all empirical intuitions in space (non datur hiatus); for one can express the proposition thus: Nothing can enter experience that proves a vacuum or even permits it as a part of empirical synthesis" (B281-A229). This is wrong. From our viewpoint, Kant's discourse is untenable since we can say, in an opposite manner to what Kant refers to (A229-B282), 'as far as concerns the void that one might think of inside of the field of possible experience (the world), this belongs to the jurisdiction of the understanding, which only decides about questions concerning the use of given appearances or disappearances for empirical cognition, and it is not a problem for ideal reason, which 
goes beyond the sphere of a possible experience and would judge about what surrounds and bounds this, and must therefore not be considered in the transcendental dialectic.'

Acknowledging the above facts, we will deal with the issue of extensive magnitude in space again. We have already clarified that 1) space-time itself is cognized as nullity in space-time, through empirical intuition through which the extensive magnitude is cognized; and 2) space-time itself and extensive magnitude are homogeneous with quantum continuum. Since extensive magnitude concerning the parts and the whole signify nullity, the representation of the parts is in conformity with the representation of the whole, indicating no successive order for the two. In view of the fact that, in Kant's metaphysics, representation of space or time is homogeneous with "an a priori intuition" (B40) or a pure inner intuition, we think that Kant himself has already made a similar remark to us, saying, "Since the mere intuition in all appearances is either space or time, every appearance as intuition is an extensive magnitude, as it can only be cognized through successive synthesis (from part to part) in apprehension" (A163-B204). We have already clarified: 1) every appearance can be comprehended, through empirical intuition and the anticipation of perception, as filled space-elapsing time or empty space-nullified time: 2) space-time itself - nullity in space-time - permeates all appearances. Therefore, we partly agree with Kant, thinking that since the parts themselves signify nullity in space-time, extensive magnitude, which is cognized through empirical intuition and "successive synthesis (from part to part) in apprehension," is to be homogeneous with nullity in space-time. Since the endlessly continuing addition of nullity in space-time is already nullity in space-time, "the absolute whole of magnitude (the world-whole)" (A483) is commensurate with nullity in space-time. Therefore, we think that what Kant refers to concerning this issue: "The absolute whole of magnitude (the world-whole), of division, of descent, of the conditions of existence in general, together with all the questions about whether these are to come about through a finite or an endlessly continuing synthesis, has nothing to do with any possible experience" (A483), is absolutely wrong. Contrarily, we say that "the absolute whole of magnitude (the world-whole)" has everything to do with the possible experience of death itself - nullity in space-time - since both signify nullity in space-time. Following what Kant refers to (A518/B546-A519/B547), we should add, in an opposite manner, saying that 'in regress to the unconditioned magnitude of the world-whole in time and in space, this unbounded ascent can be called a regress to infinity. Now we have the world-whole in concept as nullity in space-time, and its possibility in intuition. Thus we can infer from its magnitude to the magnitude of the regress, and determine the latter according to the former.'

In relation to the issue of the manifold of the homogeneous, Kant elaborates on sensation and magnitudes, saying, "Now since sensation in itself is not an objective representation, and in it neither the intuition of space nor that of time is to be encountered, it has, to be sure, no extensive magnitude, but yet it still has a magnitude (and indeed through its apprehension, in which the empirical consciousness can grow in a certain time from nothing $=0$ to its given measure), thus it has an intensive magnitude, corresponding to which all objects of perception, insofar as they contain sensation, must be ascribed an intensive magnitude, i.e., a degree of influence on sense" (B208). We think that Kant's remarks indicate three things: 1) Sensation does not have extensive magnitude since it does not contain the intuition of space or that of time, while empirical intuition (i.e., pure intuition affected by the sensation of nullity) does have it: 2) the empirical consciousness grows in a certain time, through apprehension, from nothing $=\mathrm{o}$ to its given measure as an intensive magnitude; and 3) an intensive magnitude, corresponding to the degree of influence on sensation, pertains to all objects of perception. Here, what is the difference between extensive and intensive magnitudes? We think that there is no difference in empirical intuition through which both magnitudes are cognized. According to Kant, when sensation is alienated from intuition of space or of time, it can have intensive magnitude, through apprehension, "in which the empirical consciousness can grow in a certain time from nothing $=0$ to its given measure" (B208). This discourse implies that sensation, allied by apprehension, can yield intensive magnitude by bypassing empirical intuition. While intensive magnitude is not thought to emerge in "pure a priori cognition" (A102), Kant seems to think that sensation, accompanied by intuitius originarius (B72) and apprehension, is equivalent to "pure apperception," which, as "the thoroughgoing identity of oneself in all possible representations, grounds empirical consciousness a priori" (A116), yielding "a principle of the synthetic unity of the manifold in all possible intuition" (A116-A117). Since "the thoroughgoing identity of ourselves with regard to all representations," is the ground on which "empirical consciousness a priori" rests, it is "a necessary condition of the possibility of all representations" (A116). This is thought to be the ground for "the systematic unity of the manifold of empirical cognition" (A671/B699) on which "the transcendental deduction of all the ideas of speculative reason" - as "regulative principles" - is to be 
cultivated and corrected (A671/B699). These principles are "the function, unrestricted by any sensible condition" (B224). Kant's way of thinking is wrong. We have to think that "the thoroughgoing identity of oneself in all possible representations," which "grounds empirical consciousness a priori," signifies the representation 'I think' (B132), which comprises empirical intuition and apprehension. The apprehension, in regard to sensation, which Kant harbors a priori, forces him to firmly believes that "sensation in itself is not an objective representation, and in it neither the intuition of space nor that of time is to be encountered" (B208). Since the real in appearance (realitas phaenomenon) is "that in the empirical intuition which corresponds to the sensation" (A168), it signifies sensation's combination with matter (A20) - space-time itself. Therefore, in an opposite manner to what Kant refers to (A175), we have to say 'it is not false to assume that the real in appearance, as sensation's combination with space-time itself, is always equal in degree and does not differ in aggregation or its extensive magnitude, when this is asserted on the basis of principles of understanding a priori.' In relation to this issue, when Kant elaborates on boundaries (points and instances), saying "The property of magnitude on account of which no part of it is the smallest (no part is simple) is called their continuity. Space and time are quanta continua, because no part of them can be given except as enclosed between boundaries (points and instants), thus only in such a way that this part is again a space and a time. Space therefore consists only of spaces and time of times. Points and instances are only boundaries, i.e., mere places of their limitation; but places always presuppose those intuitions that limit or determine them, and from mere places, as components that could be given prior to space or time, neither space nor time can be composed" (B211-A170), we partly agree and partly disagree with Kant, thinking that 1) every part of magnitude should be nullity: 2) since space-time itself manifests itself in mere places of decease of the representation 'I think,' space and time can be composed from the mere places through empirical intuition and synthesis of the productive imagination as components that could be given prior to mere places. Therefore, in an opposite manner to what Kant refers to (A170-B212), we have to say, 'Magnitude of a space or a time can be called filled or flowing (elapsing), since the synthesis (of the reproductive imagination) in their generation is a progress in space-time, the continuity of which is customarily designated by the expression filled or elapsing.' This saying indicates that 1) space-time itself exists prior to points and instants in which empirical intuition encounters it in experience or in possible experience: 2) space-time itself bounded by points and instances signifies quanta continua: 3 ) space-time itself appears as a space and a time when it is comprehended through empirical intuition and synthesis of the productive imagination as space-time itself limited between points and instants: 4) space-time itself, limited by the boundaries, has magnitude of the sort which can be designated by the expression "filled" or "elapsing." We have already clarified that 1) all appearances themselves, "considered extensively as well as intensively, are continuous magnitudes," signify filled space-elapsing time or empty space-nullified time; and 2) space-time itself, which signifies quantum continuum, appears as quantum discretum. Therefore, when Kant makes remarks concerning the issue of sensation, degree and magnitude, saying, "every sensation has a degree or magnitude, through which it can more or less fill the same time, i.e., the inner sense in regard to the same representation of an object, until it ceases in nothingness $(=0=$ negatio). Hence, there is a relation and connection between, or rather a transition from reality to negation, that makes every reality representable as a quantum, and the schema of a reality, as the quantity of something insofar as it fills time, is just this continuous and uniform generation of that quantity in time, as one descends in time from the sensation that has a certain degree to its disappearance, or gradually ascends from negation to its magnitude" (A143-B183), we partly agree and partly disagree with Kant. We think that 1) since sensation is to combine with "the inner sense in regard to the same representation of an object" (A143), it does have "a degree or magnitude" in terms of the empirical consciousness: 2) "the quantity of something insofar as it fills time" pertains to filled space-elapsing time: 3 ) when the inner sense ceases to function in disappearance, sensation, having filled the same time with the inner sense, ceases to be with the empirical consciousness; and 4) even if sensation is detached from the empirical consciousness along with a degree or intensive magnitude, sensation combined with space-time itself lasts. This implies, in opposition to Kant who says "sensation in itself is not an objective representation" (B208), that sensation in itself, which combines with "the inner sense in regard to the same representation of an object," is an objective representation whose validity rests on the transition from sensation with a degree or intensive magnitude to sensation with no degree or extensive magnitude. Even if the inner sense and sensation with a degree or intensive magnitude cease to function, reality - sensation combined with space-time itself - does last as a quantum. We think that 1) all alteration as a transition of a thing from one state to another signifies the transition from a state of appearance to a state of disappearance, which is correspondent to a transition of space-time itself from 'filled-elapsing' to 'empty-nullified;' and 2) the form of appearance, which alterability concerns, is 'filled-elapsing' or 'empty-nullified,' while their cause is in 
the unalterable - space-time itself. When Kant adamantly opposes this kind of metaphysics, saying, "no perception, hence also no experience, is possible that, whether immediately or mediately (through whatever detour in inference one might want), would prove an entire absence of everything real in appearance, i.e., a proof of empty space or of empty time can never be drawn from experience" (B214), we have to say, in an opposite manner to what he says (B214-A173), "perception, hence also experience, is possible that, whether immediately or mediately (through whatever detour in inference one might want), would prove an entire presence of everything real in disappearance, i.e., a proof of empty space or of empty time can be drawn from experience or from possible experience. For, first, the entire presence of the real in disappearance can be perceived in possible experience of death, and, second, it can be deduced from any single disappearance, from the difference in the degree of reality, and it may be assumed for the explanation of that. If the entire intuition of a determinate space or time is real through and through, every part of it is empty or filled.' Then, we agree with Kant, who says, "since every reality has its degree that can decrease to nothing (emptiness) through infinite steps while the extensive magnitude of the appearance remains unaltered, it must yield infinitely different degrees with which space or time is filled, and the intensive magnitude in different appearances can be smaller or greater even though the extensive magnitude of the intuition remains identical" (B214-A173). Here, we have to note that 1) sensation which has a degree or intensive magnitude is to be altered into sensation which has no degree or extensive magnitude only upon ceasing in nullity; 2) "every reality" which has "its degree that can decrease to nothing (emptiness) through infinite steps" signifies quantum discretum.

In regard to the commencement of synthesis of apprehension in the standing and lasting I, Kant explicates it himself (A499). In view of the discourse we have made so far, we should say, in an opposite manner, 'the appearances, in their apprehension, are themselves nothing other than an empirical synthesis in space-time itself, and thus are given only in this synthesis. Since the conditioned (in appearance) is given as appearance which is to disappear, the synthesis constituting $a$ priori condition is thereby also given and presupposed. This synthesis need not take place for the first time in the regress, and without it. And in such a case one can very well say that a regress to the conditions, i.e., a continued empirical synthesis on this side is not demanded or is not given as a problem,...' Furthermore, in regard to the issue of the "anticipation of perception," Kant makes convoluted, astonishing remarks, saying, "The quality of sensation is always merely empirical and cannot be represented a priori at all (e.g. colors, taste, etc.). But the real, which corresponds to sensations in general, in opposition to the negation $=0$, only represents something whose concept in itself contains a being, and does not signify anything except the synthesis in an empirical consciousness in general. In inner sense, namely, the empirical consciousness can be raised from o up to any greater degree, so that the very same extensive magnitude of intuition (e.g., an illuminated surface) can excite as great a sensation as an aggregate of many other (less illuminated) surfaces taken together. One can therefore abstract entirely from the extensive magnitude of appearance and yet represent in the mere sensation in one moment a synthesis of uniform increase from o up to the given empirical consciousness" (B217-A176-B218). We partly agree with Kant. The most important thing here is how "one can therefore abstract entirely from the extensive magnitude of appearance and yet represent in the mere sensation in one moment a synthesis of uniform increase from o up to the given empirical consciousness." From our viewpoint, Kant's discourse seems to testify that Kant has made a mistake, which forced him to fabricate a thing in itself, which is supposedly an epistemological naught. Why? It is clear that the standing and lasting $\mathrm{I}$, which is supposed to have the "anticipation of perception" (B217), cannot sense, intuit or cognize "a priori in magnitudes in general only with a single quality, namely continuity" without encountering it in experience or in possible experience. When the standing and lasting I necessarily and universally encounters disappearance of "the manifoldness of space and time" (A107) in experience or in possible experience, and senses nullity in space-time, "the real, which corresponds to sensations," "a single quality" in magnitude, namely "continuity" and "intensive quantity" in "the real of appearances" are all given a priori. We have to note that since a human perceives "the real, which corresponds to sensations in general, in opposition to the negation $=0$ " or in conformity with the negation $=0$, "what pertains to sensation" manifests itself to "represent something whose concept in itself contains a being," (B217) and "does not signify anything except the synthesis in an empirical consciousness in general" (B217-A176). Appearances themselves signify filled space-elapsing time or nullity in space-time that is given a priori. Since this does not constitute "the real difference between empirical and a priori cognition," but constitutes "the sensation (as matter of perception)" (A167-B209) - quantum, it follows that it is really only this quantum - "the sensation (as matter of perception)" - that can be anticipated at all. Therefore, this would not "deserve to be called an anticipation in an unusual sense," since it seems natural "to 
anticipate experience precisely in what concerns its matter, which one can draw out of it" (B209) as "unbounded reality" - "the matter of all possibility" (B322). When the standing and lasting I of pure apperception could acquire the concept of death - the pure concepts of understanding - through empirical intuition and synthesis of apprehension, upon encountering the phenomena of disappearance, it emerged as a human. In other words, when it cognized nullity in space-time, which already resides in them, it emerged in the representation of space-time itself, and began to exist as a human.

In association with the issue of magnitude, Kant has made an important remark, saying "from the empirical consciousness to the pure consciousness a gradual alteration is possible, where the real in the former entirely disappears, and a merely formal (a priori) consciousness of the manifold in space and time remains; thus there is also possible a synthesis of the generation of the magnitude of a sensation from its beginning, the pure intuition $=0$, to any arbitrary magnitude" (B208). The remark gives us a hint on how a human cognizes the magnitude in space-time through empirical intuition and synthesis, making it possible for us to extend it to the furthest point as far as we can think. We think that what Kant refers to here indicates four things: 1) When the empirical consciousness, namely a standing and lasting I disappears, the real appears to be lost: 2) when the real in the empirical consciousness appears to be lost, what remains is the pure consciousness, namely "a merely formal ( $a$ priori) consciousness of the manifold in space and time," which signifies unbounded reality as the matter of all possibility (B322): 3) a merely formal (a priori) consciousness, as unbounded reality, cognizes magnitude through the "synthesis of the generation of the magnitude of a sensation," which begins in "the pure intuition $=0$;" and 4) this magnitude can expand to "any arbitrary magnitude." We think that "the pure consciousness," or "a merely formal (a priori) consciousness," signifies the dismembered standing and lasting I of pure apperception, which is to occur in experience or in possible experience, and "the pure intuition $=0$ " signifies nullity in space-time, indicating that Kant's metaphysics agree with our discourse concerning space-time itself and magnitudes, except that the alteration "from the empirical consciousness to the pure consciousness," namely dissolution of the empirical consciousness, is to be precipitous and irreversible. Furthermore, when Kant makes a remark in regard to the issue of the representation 'I think, saying, "The 'I think' must be able to accompany all my representations; for otherwise something would be represented in me that could not be thought at all, which is as much as to say that the representation would either be impossible or else at least would be nothing for me. That representation that can be given prior to all thinking is called intuition. Thus all manifold of intuition has a necessary relation to the 'I think' in the same subject in which this manifold is to be encountered. But this representation is an act of spontaneity, i.e., it cannot be regarded as belonging to sensibility. I call it the pure apperception, in order to distinguish it from the empirical one, or also the original apperception, since it is that self-consciousness which, because it produces the representation 'I think,' which must be able to accompany all others and which in all consciousness is one and the same, cannot be accompanied by any further representation. I also call its unity the transcendental unity of self-consciousness in order to designate the possibility of a priori cognition from it" (B131-B132), we agree with him, thinking that pure apperception "cannot be accompanied by any further representation" than "a necessary relation." The most important thing is what this "necessary relation" means. Our transcendental analytic indicates that 1) all manifold of intuition contains "a necessary relation," which is space-time itself: 2) this manifold is encountered in the same subject - the 'I think,' namely filled space-elapsing time: 3) the representation of "a necessary relation" - "pure apperception" - signifies space-time itself: 4) "a necessary relation" manifests itself in the entire dissolution of the "unity of self-consciousness" - death - which occurs necessarily and universally; and 5) the dissolution of the "unity of self-consciousness' is "an act of spontaneity." Since the representation 'I think' - the transcendental unity of self-consciousness - is homogeneous with "pure apperception" which signifies "the thoroughgoing identity of oneself in all possible representations" which "grounds empirical consciousness a priori" (A116), our metaphysics implies that the representation 'I think,' which can accompany all others, is to cognize "through categories whatever objects may come before our senses" (B159). In this context we comprehend that a human, as the representation 'I think,' senses, intuits and cognizes all appearances themselves in virtue of filled space-elapsing time or nullity in space-time through empirical intuition and synthesis. Thus, we rescue Kant's metaphysics from the abyss of emptiness, in which he does not comprehend something, which would be represented in him as possibility.

Nevertheless, Kant's ultimate aim - guarding the purity of pure reason on which the transcendental concept of reason and a pure category rest (YAMAMOTO 2016: 87-100), would command him not to accept this rescue. Kant divulges the deepest motif of his metaphysics, saying, "The ground of the regulative principle of reason is the proposition that in the empirical regress there can be encountered no experience of an absolute boundary, and hence no experience of a condition as one that is 
absolutely unconditioned empirically" (A517/B545). We say, in an opposite manner, 'in the empirical regress, there can be encountered possible experience of an absolute boundary, and hence possible experience of a condition as one that is absolutely unconditioned empirically. The reason for this is that such a possible experience would have to contain, in itself, a bounding of appearance by nullity, or by the void, which the regress, even if not carried on far enough, would encounter by means of a perception - which is possible.' Therefore, we think that there is little ground for the regulative principle of reason, but there is ample ground for the constitutive principle of reason. Our transcendental analytic has shown that 1) "the absolute unity of the thinking subject" and "the absolutely unconditioned in a series of given conditions" (A335) signify nullity in space-time quantum; and 2) "a being of all beings" (A336) signifies space-time itself - quantum. Following what Kant says (A246/B303-A247), we say, "That the understanding can never accomplish a priori anything more than to anticipate an object of experience or possible experience in general, and, since that which is not appearance itself cannot be an object of experience or possible experience, it can never overstep the limits of sensibility, within which alone objects in themselves are given to us.' Then, we should like to say, in an opposite manner to what Kant refers to (B139), 'the human understanding can form for itself the concept of another possible understanding, either one that would intuit itself, or one that, while possessing a sensible intuition, would possess one of a different kind than one grounded in space and time.' The "concept of another possible understanding" (B139) seems to be homogeneous with Kant's thing in itself.

\section{REFERENCES}

Kant, Immanuel, Critique of Pure Reason, Cambridge University Press, 1999.

Yamamoto, Kazuhiko, "The Pure Concepts of the Understanding and Synthetic A Priori Cognition: the Problem of Metaphysics in the Critique of Pure Reason and A Solution," Proceedings of the European Conference on Ethics, Religion \& Philosophy 2016, pp 87-100, 2016

\section{AUTHOR's BIOGRAPHY}

\section{Kazuhiko Yamamoto, MD, PhD}

1997 - 2003 Professor, Kyushu Institute of Design

2003 - 2017 Professor, Kyushu University 